\title{
Low Expression of miR-491 Promotes Esophageal Cancer Cell Invasion by Targeting TPX2
}

\author{
Hongyan Niu Li Gong $^{\mathrm{b}}$ Xiaofeng Tian ${ }^{\mathrm{b}} \quad$ Liangwei Fang $^{\mathrm{b}} \quad$ Chengxiang Wang ${ }^{\mathrm{b}}$ \\ Yi Zhu
}

aDepartment of Clinical Laboratory, Huai'an Hospital Affiliated of Xuzhou Medical College and Huai'an Second People's Hospital, Huai'an, 'Department of Thoracic and Cardiovascular Surgery, Huai'an Hospital Affiliated of Xuzhou Medical College and Huai'an Second People's Hospital, Huai'an, China

\section{Key Words}

MiR-491 • TPX2 • EMT • Invasion • Esophageal cancer

\begin{abstract}
Background/Aims: MicroRNAs (miRNAs) have a key role in carcinogenesis and cancer development, but the role of miRNAs in the progression of esophageal cancer (EC) remains unclear. Methods: The TE-1 and Eca-109 EC cell lines were used. The expression of miR-491 and candidate gene TPX2 in EC samples $(n=99)$ were detected by RT-PCR. The cells invasive ability was determined by transwell assay. The luciferase reporter assay was used to confirm the regulation mechanism. Results: A decreased expression of miR-491 was detected in the EC clinical samples compared with the corresponding adjacent tumor tissues. Aberrant expression of miR-491 regulated cells invasion and EMT markers. Furthermore, we verified that TPX2 was a target gene of miR-491. Conclusions: miR-491 may play a critical role in EC.
\end{abstract}

Copyright (C) 2015 S. Karger AG, Basel

\section{Introduction}

Esophageal cancer (EC) is the sixth leading cause of cancer mortality and the eighth most common cancer in the world [1]. According to its pathological characteristics, esophageal cancer has two main histological subtypes, esophageal squamous cell carcinoma (ESCC) and esophageal adenocarcinoma (EAC) [2]. Esophageal squamous cell carcinoma (ESCC) predominates in China, with a proportion of more than $90 \%$ of all EC [3]. Despite the application of radical esophagectomy and chemoradiotherapy, the overall 5-year survival

Yi Zhu

KARGER 125
Department of Thoracic and Cardiovascular Surgery, Huai'an Hospital Affiliated of Xuzhou Medical College, Huai'an Second People's Hospital Huai Hai South Road, Huai'an, 223001 (China); Tel. +86 517 80870120, E-Mail zhuyi0512@126.com 
rate of patients for EC is approximately 10-41\% [4]. Recent studies reported that some oncogenic and tumor suppressive factors contribute into the development of EC; however, some of them were not conclusive $[5,6]$.

miRNAs, a class of small non-coding RNAs of 20-22 nucleotides, are involved in multiple biological processes, such as cell differentiation, proliferation, oncogenesis, angiogenesis and cell invasion $[7,8]$. Accumulating evidence has shown that miRNAs can act either as oncogenes or as tumor suppressors in EC $[9,10]$. Here, functions of miR-491 related to cancers have been shown in recent studies. For example, miR-491 induced apoptosis by regulation of Bcl-XL, anti-apoptotic Bcl-2 family, commonly overexpressed in colorectal cancer [11]. miR-491 is involved in metastasis of hepatocellular carcinoma (HCC) by inhibition of matrix metalloproteinase and epithelial to mesenchymal transition [12]. Liu et al. found that miR491 was decreased in the EC tissues compared with those in the paracancerous normal tissues by using miRNA array [13]. However, the potential mechanism of miR-491 and the progression of EC were not mentioned.

Targeting protein for Xenopus kinesin-like protein 2 (TPX2) impacts spindle assembly in human cells [14]. Increasing studies showed that TPX2, acts as an oncogene, and is involved in the progression of tumors. TPX2 knockdown suppressed hepatocellular carcinoma cell invasion via inactivating AKT signaling and inhibiting MMP2 and MMP9 expression [15]. TPX2 regulates tumor growth in human cervical carcinoma cells [16]. siRNA/TPX2 could inhibit the invasion and metastasis of EC cells, and may become a new approach for treatment of esophageal carcinoma [17].

In this study, we discovered that miR-491 is reduced in EC tissues, and confirmed a functional role for miR-491 in tumor metastasis of esophageal cancer. Furthermore, our data suggested that miR-491 could regulate the invasion of EC cells by targeting TPX2.

\section{Material and Methods}

\section{Clinical Samples}

All EC tissues and the corresponding adjacent esophageal tissues were obtained from patients who had undergone routine surgery at Huai'an Hospital Affiliated of Xuzhou Medical College, between November 2009 and June 2013. All samples ( $\mathrm{n}=99$ ) were collected, immediately snap frozen in liquid nitrogen, and stored at $-80^{\circ} \mathrm{C}$ until RNA extraction. Our study was approved by the Ethical Committee of Huai'an Hospital Affiliated of Xuzhou Medical College, and all patients had written informed consent.

\section{Cell culture}

The human EC cell lines (Kyse140, TE-1, EC18, Eca-109, and HKESC1) and normal esophageal cell (NEEC) were purchased from the Institute of Biochemistry and Cell Biology of the Chinese Academy of Sciences (Shanghai, China). All the cell lines were cultured in RPMI-1640 medium with 10\% fetal bovine serum (Invitrogen, Carlsbad, $\mathrm{CA}$ ) and penicillin $(100 \mathrm{U} / \mathrm{ml})$ at $37^{\circ} \mathrm{C}$ with $5 \% \mathrm{CO}_{2}$.

\section{Isolation of total RNA and Quantitative RT-PCR}

Total RNA from tissue samples and cell lines were harvested using the TRIzol (Invitrogen, USA). The expression level of miRNAs was detected using Taqman MicroRNA Assay (Applied Biosystems) specific for hsa-miR-491.10 ng of total RNA was reverse transcribed to cDNA with specific stem-loop RT primers. All the primers were obtained from the TaqMan miRNA Assays. U6 small nuclear RNA was used for normalization. Relative expression levels of E-cadherin, N-cadherin, Vimentin and TPX2 mRNA were examined by SYBR Green real-time PCR (RT-PCR) and normalized to GAPDH. RT-PCR was performed by using the ABI 7500 Fast Real-Time PCR system (ABI, CA, USA).

\section{Cell-Proliferation Assay (Cell-Counting Kit-8)}

TE-1 and Eca-109 cells were seeded into 96-well plates $\left(6.0 \times 10^{3}\right.$ cells per well). Cell viability was investigated by cell-counting kit-8 assay (Beyotime Institute of Biotechnology, Shanghai, China). The absorbance of each well was shown on a spectrophotometer (Thermo) at $450 \mathrm{~nm}$. 


\section{Cellular Physiology Cell Physiol Biochem 2015;36:2263-2273 \begin{tabular}{l|l|l}
\hline DOI: 10.1159/000430190 & C 2015 S. Karger AG, Basel
\end{tabular} and Biochemistry Published online: July 24, 2015 \\ Niu et al.: Low Expression of miR-491 Promotes Cell Invasion}

\section{Transwell assay}

According to the manufacturer's protocol, Matrigel-coated invasion chambers ( $8 \mu \mathrm{m}$, Costar) were used to explore the cells invasion. Briefly, miR-491 mimic or miR-491 inhibitor transfected cells were harvested, resuspended $\left(1 \times 10^{5}\right.$ cells/well $)$ in 200- $\mu \mathrm{l}$ serum-free medium, and transferred to the upper chamber of the Matrigel-coated inserts; culture medium containing $10 \%$ FBS was placed in the bottom chamber. The cells were incubated for $24 \mathrm{~h}$ at $37^{\circ} \mathrm{C}$. After incubation, the noninvaded cells on the upper membrane surface were removed with a cotton tip, and the cells that passed through the filter were fixed and stained using $0.1 \%$ crystal violet. The results were shown by counting three fields per membrane and represented the average of three independent experiments.

\section{Western blot}

Western blotting was performed according to standard protocols. All proteins were extracted from tissues or cultured cells using RIPA buffer containing PMSF (Roche, Basel, Switzerland), quantities using a protein assay (BCA method, Beyotime, China). And total proteins were fractionated by sodium dodecyl sulfate polyacrylamide gel electrophoresis (SDS-PAGE) transferred to polyvinylidene fluoride (PVDF) membrane, blocked in 5\% dry milk for 1 hour and immunostained with antibodies at $4^{\circ} \mathrm{C}$ overnight using anti-TPX2, anti-E-cadherin, anti-N-cadherin, anti-Vimentin (1:500, Santa Cruz, USA) and anti-GAPDH (1:5000, kangcheng, China). The integrated density of the band was quantified by Image J software (NIH). The values were read through a chemiluminescent detection system (Pierce ECL Substrate Western blot detection system, Thermo, Pittsburgh, PA) and exposed in Molecular Imager ChemiDoc XRS System (BioRad, Hercules, CA).

\section{Transient transfection}

According to the instructions provided by the manufacturer, the cells were seeded into 6-well plates. Oligonucleotides NC (control for miR491-mimics), mimics (hsa-miR-491), inhibitor NC (control for miR-491 inhibitor), inhibitor (hsa-miR-491 inhibitor) was purchased from GenePharma (Shanghai, PR China). The transfection was conducted by using Lipofectamine 2000 Reagent (Invitrogen, Carlsbad, CA, USA) at a final concentration of $100 \mathrm{nM}$. Cells were transfected with $50 \mathrm{nM}$ siRNA/TPX2 and siRNA/control (GenePharma, Shanghai, China) using Lipofectamine 2000 (Invitrogen, USA).

\section{Plasmid construction and cell transduction}

The predicted target sites of 3'-UTR of human TPX2 gene or a mutant sequence with the predicted target sites was inserted into the KpnI and SacI sites of pGL3 promoter vector (Genscript, Nanjing, China). By using Lipofectamine 2000 (Invitrogen Corp, CA, USA), all the cells were plated onto 6-well plates and were transfected with $100 \mathrm{ng}$ of pGL3-TPX2 or pGL3-TPX2-MUT, and miR-491 mimics (50nM). TPX2 gene was synthesized (purchased from Genscript, Piscataway, NJ) with restrictive digestion using Mlu I and subcloned pLV-GFP plasmid, it was named pLV-GFP-TPX2. All cells were transfected with lentivirus using polybrene $(8 \mathrm{ug} / \mathrm{ml})$.

Luciferase activities analysis

According to the manufacturer's protocol, luciferase actions were measured in Victor 1420 Multilabel Counter (Wallac, Finland) using Luciferase Assay System (Promega, USA).

\section{Statistical methods}

In this study, the method of 2- $\Delta \mathrm{Ct}$ was used to detect the results of RT-PCR. All statistical analyses were performed using STATA 9.2, and presented with Graph Pad Prism software. Data are expressed as the mean \pm SD from at least three separate experiments. Data were analyzed using Student's $t$ test or one-way ANOVA. Values of $\mathrm{P}<0.05$ were considered statistically significant.

\section{Results}

Expression of miR-491 is reduced in EC tissues

First, the expression level of miR-491 was examined by quantitative reverse transcription-PCR (qRT-PCR) in both EC tissues and adjacent specimen. As shown in Fig. 1A KARGER 
and $\mathrm{B}$, we found that miR-491 expression was down-regulated and TPX2 was up-regulated in EC tissues ( $\mathrm{n}=99)$. Total EC patients were divided into two groups using median mRNA level of miR-491 ( ${ }^{a}$ median $=0.3043$ ) and TPX2 ( ${ }^{b}$ median=3.0027) as threshold, respectively. The correlation between miR-491 (TPX2) expression and clinicopathological characteristics shown that there was markedly related to tumor stage $(\mathrm{p}<0.000)$ and metastasis $(\mathrm{p}<0.000)$ (Table 1). In addition, we discovered that the expression of miR-491 was inversely correlated with TPX2 expression ( $R=-0.827 ; p<0.0001$; Fig. $1 \mathrm{C})$ in cancer samples. Thus, we speculated that miR-491 might be a critical tumor suppressor gene in EC.

\section{MiR-491 regulates EC cell invasion but had no effect on cell proliferation}

In our study, the expression of miR-491 was examined in five EC cell lines (Kyse140, TE1, EC18, Eca-109, and HKESC1) and a normal esophageal cell line (NEEC) by using qRT-PCR. As shown in Fig 2A, the expression of miR-491 was reduced in all five EC cell lines, compared with the normal NEEC cell line. Here, the TE-1 and Eca-109 EC cell lines were chosen to investigate the effect of miR-491. Eca-109 and TE-1 cell lines were transfected with NC, miR491 mimics, inhibitor NC and miR-491 inhibitor respectively. The qRT-PCR assay was used to confirm the transfection efficiency (Fig. 2B). The cell viability was examined using the CCK8

Table 1. Expression levels of miR-491 and TPX2 in EC and corresponding adjacent tissues. AC: Adenocarcinoma, SCC: Squamous cell carcinoma. ${ }^{\mathrm{a}}$ median $=0.3043,{ }^{\mathrm{b}}$ median $=3.0027$. *indicates $\mathrm{p}<0.05$

\begin{tabular}{|c|c|c|c|c|c|c|c|}
\hline Characteristics & $\begin{array}{c}\text { All } \\
\text { Patients }\end{array}$ & $\begin{array}{l}\text { miR-491 low } \\
\text { expression } \\
\left(\leq \text { Median }^{\mathrm{a}}\right)\end{array}$ & $\begin{array}{c}\text { miR-491 high } \\
\text { expression } \\
\left(>\text { Median }^{\mathrm{a}}\right)\end{array}$ & $\underset{\text { value }}{\mathrm{p}}$ & $\begin{array}{l}\text { TPX2 low } \\
\text { expression } \\
\left(<\text { Median }^{\mathrm{b}}\right) \\
\end{array}$ & $\begin{array}{l}\text { TPX2 high } \\
\text { expression } \\
\left(\geq \text { Median }^{\mathrm{b}}\right) \\
\end{array}$ & $\begin{array}{c}\mathrm{p} \\
\text { value }\end{array}$ \\
\hline $\mathrm{n}$ & 99 & 50 & 49 & & 49 & 50 & \\
\hline Age(year) & & & & 0.472 & & & 0.753 \\
\hline$<64$ & 44 & 24 & 20 & & 21 & 23 & \\
\hline$\geq 64$ & 55 & 26 & 29 & & 28 & 27 & \\
\hline Gender & & & & 0.622 & & & 0.934 \\
\hline Male & 59 & 31 & 28 & & 29 & 30 & \\
\hline Female & 40 & 19 & 21 & & 20 & 20 & \\
\hline Histology & & & & 0.925 & & & 0.619 \\
\hline $\mathrm{AC}$ & 46 & 23 & 23 & & 24 & 22 & \\
\hline SCC & 53 & 27 & 26 & & 25 & 28 & \\
\hline Tumor depth & & & & $0.033^{*}$ & & & $0.011^{*}$ \\
\hline Tis,T1 & 40 & 15 & 25 & & 26 & 14 & \\
\hline $\mathrm{T} 2, \mathrm{~T} 3, \mathrm{~T} 4$ & 59 & 35 & 24 & & 23 & 36 & \\
\hline Stage & & & & $0.000^{*}$ & & & $0.000^{*}$ \\
\hline $0, \mathrm{I}$ & 39 & 10 & 29 & & 28 & 11 & \\
\hline II , III,IV & 60 & 40 & 20 & & 21 & 39 & \\
\hline Metastasis & & & & $0.000^{*}$ & & & $0.000^{*}$ \\
\hline No & 59 & 19 & 40 & & 39 & 20 & \\
\hline Yes & 40 & 31 & 9 & & 10 & 30 & \\
\hline
\end{tabular}

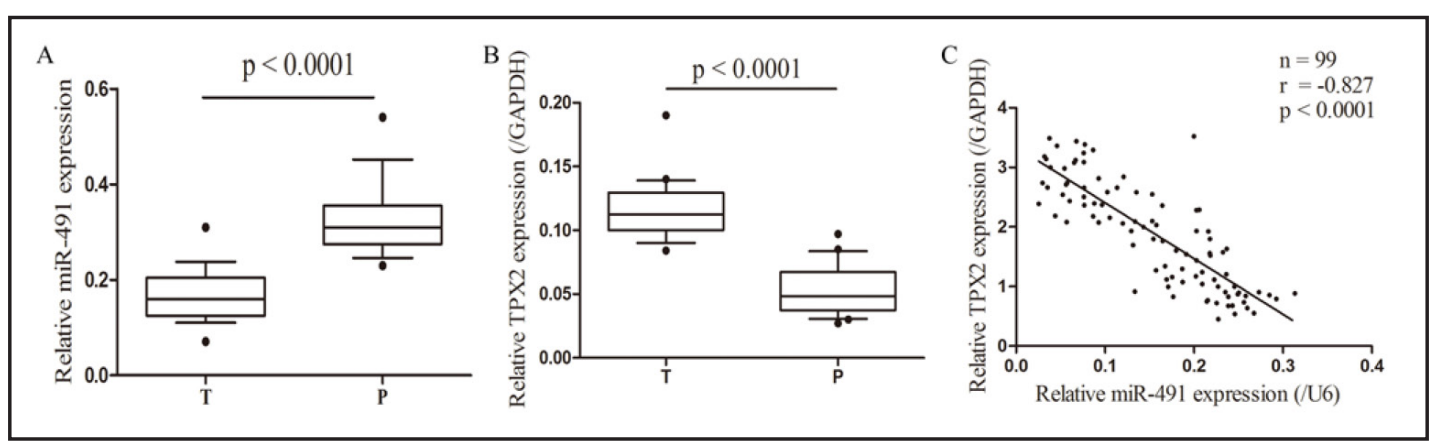

Fig. 1. Expression levels of miR-491 and TPX2 in human EC tissues. A and B: Relative miR-491 and TPX2 mRNA expression levels in human EC tissues (T) and corresponding adjacent tissues (P) relative to U6 were determined by qRT-PCR analysis. ( $\mathrm{n}=99$, $\mathrm{p}<0.0001)$. U6 small nuclear RNA was used for normalization. C: A negative correlation was found between RNA expression of miR-491 and TPX2 in tumor samples $(\mathrm{R}=-0.827 ; \mathrm{P}<0.0001)$. Data are represented as mean \pm SEM.

\section{KARGER}




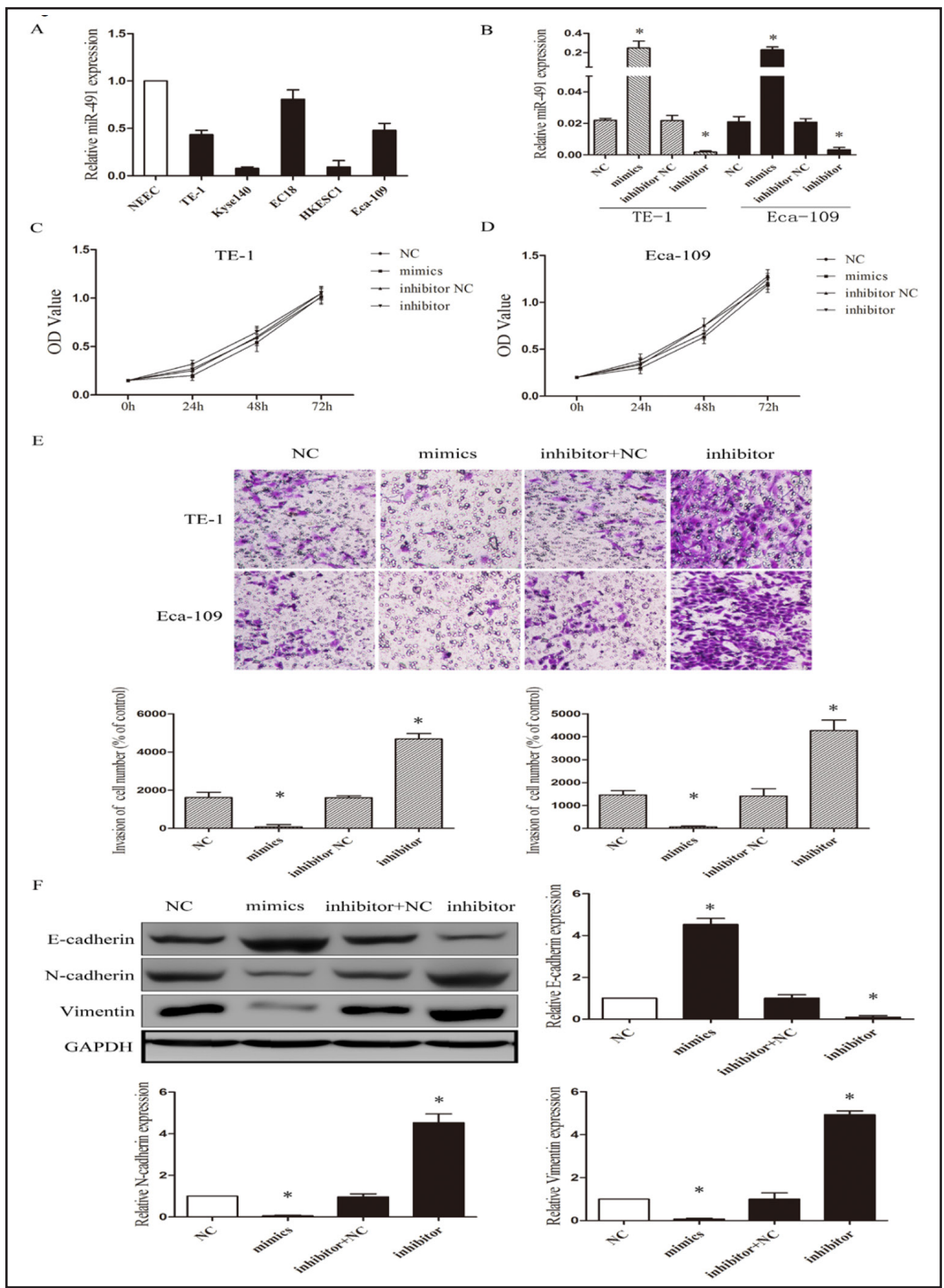

Fig. 2. miR-491 regulates EC cells invasion but has no effect on cell proliferation. A: Real-time PCR analysis of miR-491 expression in normal esophageal cells (NEEC) and esophageal cancer cell lines, including Kyse140, TE-1, EC18, Eca-109, and HKESC1. B: miR-491 expression level in cell lines transfected with NC (control for miR491-mimics), mimics (hsa-miR-491), inhibitor NC (control for miR-491 inhibitor), and inhibitor (hsa-miR-491 inhibitor). The result was confirmed by real-time PCR. C and D: The cell-proliferation assay test showed no difference on proliferation after manipulation of miR-491 in both TE-1 and Eca-109 cells at 24-hour, 48-hour, and 72-hour time points. E: Transwell assay was performed as shown in Materi- 
als and Methods. The representative images of invasive cells at the bottom of the membrane stained with crystal violet were visualized as shown. The quantifications of cell invasion were presented as percentage of cell numbers. F: The protein expression levels of E-cadherin, N-cadherin and Vimentin in Eca-109 cells transfected with NC (control for miR491-mimics), mimics (hsa-miR-491), inhibitor NC (control for miR-491 inhibitor), and inhibitor (hsa-miR-491 inhibitor) were measured by using western-blotting assay. GAPDH was used to serve as the loading control. Average values of integrated optical density (IOD) were evaluated by analyzing and recorded in the histogram. All experiments were performed in triplicate and presented as mean \pm SEM. * indicates significant difference compared with control group $(\mathrm{p}<0.05)$.

assay to analyze the biologic function of miR-491 on cell proliferation of transfected Eca-109 and TE-1. The cell-proliferation assay test showed no distinct differences on proliferation at 24-hour, 48-hour, and 72-hour time points. The assay exhibited that downregulated or upregulated miR-491 did not regulate cell proliferation (Fig. 2C and D).

Moreover, in a cell invasion assay, we observed that compared with control group, cell invasion was significantly inhibited by transfection of miR-491 mimics, whereas the cell invasion ability was promoted by transfection of miR-491 inhibitor (Fig. 2E).These results implied that miR-491 may play a critical role in suppressing EC metastasis.

Down-regulation of miR-491 could induce EMT in vitro

Epithelial-to-mesenchymal transition (EMT) is a key element of tumor cell metastasis. EMT inhibition may be a potential way to regulate the progression of cancer metastasis. In

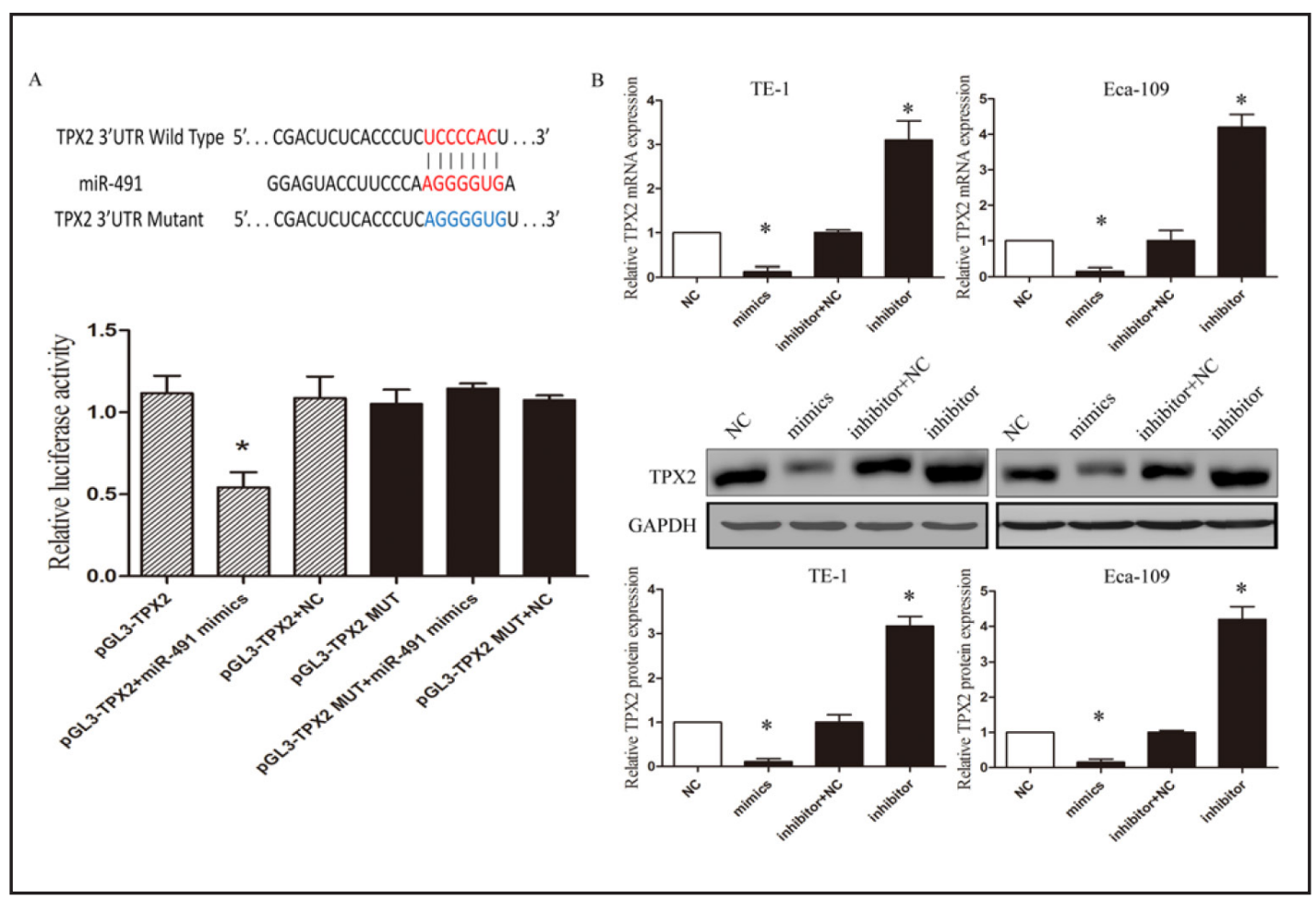

Fig. 3. miR-491 negatively regulates TPX2 expression in EC cell lines. A: The potential miR-491 seed region at the 3'-UTR of TPX2 mRNA was predicted by microRNA.org. Eca-109 cells were co-transfected with miR491 mimics (or NC) with pGL3-TPX2 (or pGL3-TPX2-MUT) vector. Luciferase activity was normalized by the ratio of firefly and Renilla luciferase signals. B: The mRNA and protein expression levels of TPX2 in TE-1 and Eca-109 cells transfected with NC, miR-491 mimics, inhibitor NC and miR-491 inhibitor were detected by using qRT-PCR and western-blotting assays. All experiments were performed in triplicate and the band intensity values were measured by using Image J. Then, the significance in change in protein expression was analyzed by using statistical t-tests. Data are represented as mean \pm SEM. * indicates $\mathrm{p}<0.05$.

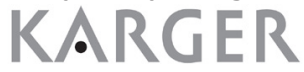




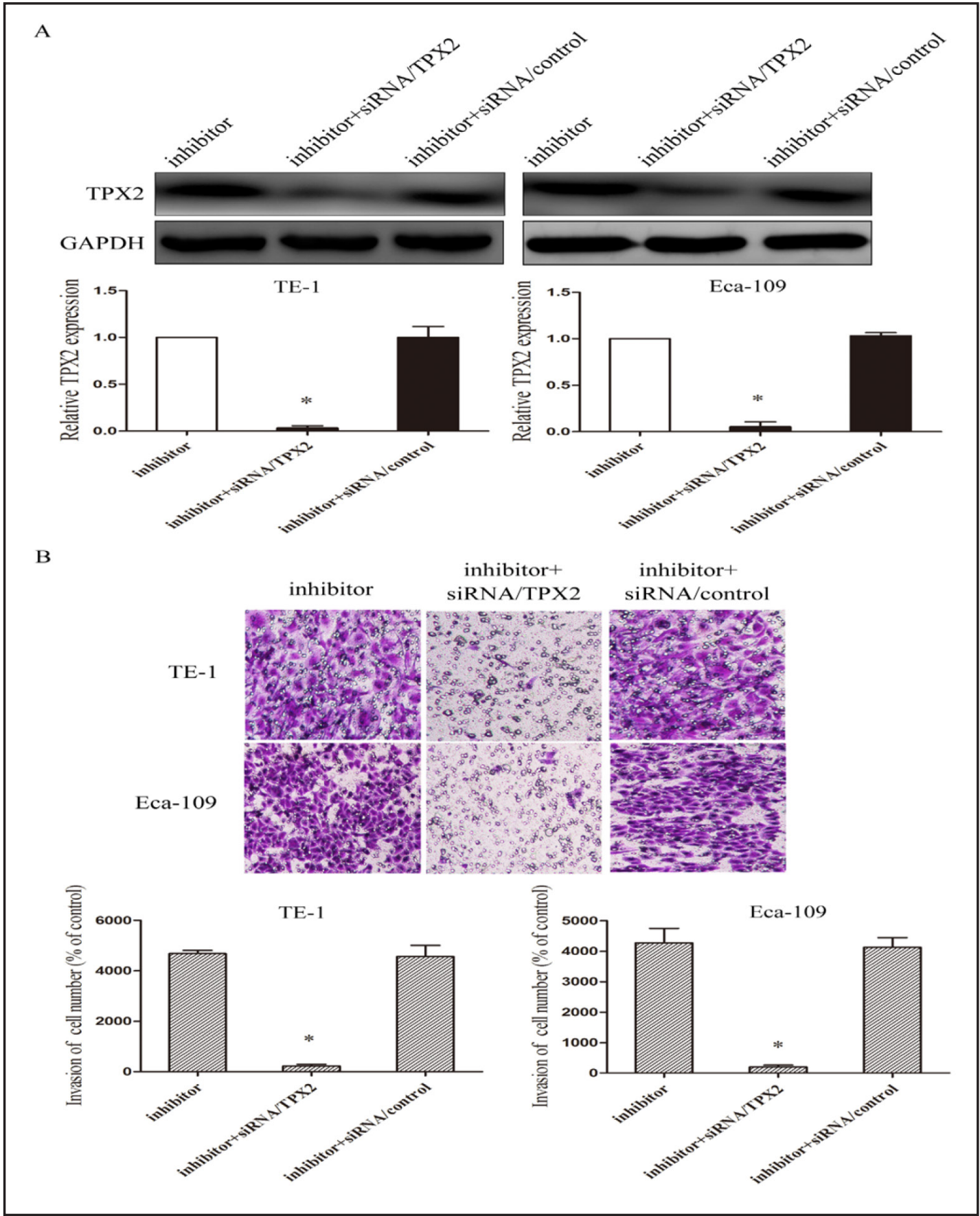

Fig. 4. Knockdown of TPX2 reverses the effect of miR-491 downregulated. A: Western-blotting assay was used to validate the transfection efficiency after both TE-1 and Eca-109 cells transfected with miR-491 inhibitor and inhibitor+siRNA/TPX2 (the co-transfection cells of miR-491 inhibitor and siRNA/TPX2) and inhibitor+siRNA/control (co-transfected with miR-491 inhibitor and siRNA/control). Average values of integrated optical density (IOD) were evaluated by analyzing five fields per slide and recorded in the histogram. B: Transwell invasion assay was utilized to analyze the effect of miR-491 on cell invasion. Cells were treated with miR-491 inhibitor and inhibitor+siRNA/TPX2 (the co-transfection cells of miR-491 inhibitor and siRNA/TPX2) and inhibitor+siRNA/control (co-transfected with miR-491 inhibitor and siRNA/control) for $24 \mathrm{~h}$. The representative images of invasive cells at the bottom of the membrane stained with crystal violet were visualized as shown. The quantifications of cell invasion were presented as percentage of cell numbers. * indicates $\mathrm{p}<0.05$. 
our study, we investigated the expression of mesenchymal markers, including N-cadherin and Vimentin and the epithelial marker, E-cadherin to determine whether miR-491 could regulate the molecular changes of EMT by using western blot assay. The western blot assay showed that up-regulated miR-491 resulted in elevated E-cadherin expression and decreased $\mathrm{N}$-cadherin and Vimentin expression. Meanwhile, down-regulated miR-491 resulted in decreased E-cadherin expression and elevated N-cadherin and Vimentin expression (Fig 2F). Taken together, the result implied that aberrant expression of miR-491 could regulate the molecular changes of EMT in EC in vitro.

TPX2 is a potential target gene of miR-491

Using publicly available algorithms named microRNA.org (http://www.microrna.org/ microrna/), miRDB (http://mirdb.org/cgi-bin/), and DIANA-MICROT (http://diana.cslab. ece.ntua.gr/micro-CDS/), we focused on TPX2. Three previous studies showed that TPX2 is an oncogene and plays an important role in ESCC progression [17-20].

To assess whether TPX2 is regulated by miR-491, luciferase reporter assay and western blot analysis were used. First, according to results of prediction, we cloned the 3'-UTR fragment containing the predicted site into pGL3 luciferase reporter vector (pGL3-TPX2). The 3'-UTR fragment with mutant sequence was also cloned as a control group (pGL3-TPX2MUT) in the predicted target site. The luciferase reporter assay revealed that the luciferase activity decreased in Eca-109 cells with miR-491 mimics and pGL3-TPX2 vectors. However, miR-491 mimics did not have any effect on luciferase activity when target cells were transfected with pGL3-TPX2-MUT vector (Fig. 3A). Next, qRT-PCR and western blot assays were performed to verify the expression level of TPX2 responses to the changes of miR-491 expression both in TE-1 and Eca-109 cell lines. As shown in Fig. 3B, the assay showed a negative regulatory effect of miR-491 on TPX2. Both in mRNA and protein expression level, up-regulated miR-491 could decrease the level of TPX2; meanwhile, down-regulated miR491 could increase TPX 2 level. These data indicated that miR-491 bound directly to specific site in the $3^{\prime}$-UTR of TPX2.

Knockdown TPX2 by siRNA partially reverses the effect of miR-491 inhibition

To further confirm whether the functional role of miR-491 on cell lines was dependent on TPX2, we also conducted a rescue experiment. Thus, the expression of TPX2 was downregulated by RNA interference in cells which were transfected with miR-491 inhibitor. All the cells were co-transfected with miR-491 inhibitor and siRNA/TPX2; meanwhile, the transfection cells of miR-491 inhibitor and the co-transfection cells of miR-491 inhibitor and siRNA/control were regarded as the control groups. The western blot assay was used to evaluate the transfection efficiency (Fig. 4A). The assay showed that TPX2 was significantly decreased both in cells co-transfected with miR-491 inhibitor and siRNA/TPX2 compared with the control groups. Furthermore, the transwell invasion assay was used to detect the cells invasive ability. The assay exhibited that the invasive ability indeed reversed to some extent when silenced TPX2 in cells transfected with miR-491 inhibitor compared with the control groups (Fig 4B). All the results indicated that the potential role of miR-491 on EC cell lines was dependent on TPX2.

\section{Discussion}

Esophageal cancer is a major cause of cancer-related death in the world and is the fourth most lethal type of cancer in China [20]. The complications arising from metastasis were the major causes of death from cancer. It is well known that altered expression of miRNAs has been implicated in the regulation of EC biological processes [21-23]. miR-16 induced the suppression of cell apoptosis while promote proliferation in esophageal squamous cell carcinoma [24]. miR-625 down-regulation promotes proliferation and invasion in esophageal 
cancer by targeting Sox2 [25]. Liu et al. [13] has performed a miRNA array analysis of EC tissues and found that miR-491 was decreased in the tumor tissues compared with those in the paracancerous normal tissues. Here, in EC, we focused on providing evidence for miR491 targeting of TPX2 to regulate the cells invasion.

An increasing number of studies demonstrated that miRNAs regulated multiple cellular pathways by affecting the expression of various target genes [26, 27]. miRNAs are considered as potential novel targets for cancer diagnosis and therapy $[28,29]$. miR-491 could effectively target both Bcl-xL and TP53 and induce cell apoptosis independent of TP53 [30]. miR-491 and GIT1 serve as modulators and biomarkers for oral squamous cell carcinoma invasion and metastasis [31]. Here, miR-491 is reduced in EC tissues. However, the potential mechanism of miR-491 in EC still remains unclear. EMT is considered to be involved in migration, tumor invasion and dissemination [32-34]. Decreased E-cadherin and elevated Vimentin and $\mathrm{N}$-cadherin expression is one hallmark of EMT which is a key element in the cancer invasion [35]. Many studies established functional associations between noncoding microRNAs and key effectors of EMT occurring in the context of carcinogenesis and embryonic development, such as miR-200 [36] and miR-10b [37]. In this study, expression of miR-491 in EC specimens was significantly lower than in corresponding adjacent tissues. Up-regulated miR-491 expression resulted in increased E-cadherin expression and decreased $\mathrm{N}$-cadherin and Vimentin expression. Meanwhile, suppressed miR-491 expression resulted in decreased E-cadherin expression and increased N-cadherin and Vimentin expression. In addition, TPX2 expression is increased in EC tissues. In human tumors, over-expression of TPX2 plays an important role in carcinogenesis and malignant progression. Especially in EC, Liu et al. had successfully silenced TPX2 gene in EC cell lines by RNAi technique. Their results showed that TPX2 siRNA could effectively inhibit the invasion and metastasis of EC cell lines, and may become a new approach for treatment of EC. In our study, we found that TPX2 is one target gene of miR-491 and miR-491 could regulate the EC development by targeting TPX2 gene.

In summary, we described, for the first time, that miR-491 was decreased in our 99 EC cases, and was related to tumor stage and metastasis. In vitro, abnormal expression of miR-491 controlled the cells invasive ability and regulated the markers of EMT in human EC cell lines. Additionally, we identified TPX2 as a direct and functional target of miR-491. Finally, we also revealed that TPX2 suppression was essential for down-regulated miR-491induced cell invasion in EC. Based on all these findings, we proposed the regulatory function of miR-491 on TPX2 by direct binding of its 3'UTR. As the limit on the number of EC tissues and cell types, further study is required to explore the underlying role of miR-491 in the progression of EC. In future, miR-491-TPX2 pathway that we studied might be used as a therapeutic approach for EC.

\section{Abbreviations}

EC (esophageal cancer); 3'-UTR: (3'-untranslated regions); qRT-PCR (Quantitative Real Time- Polymerase Chain Reaction); FBS (fetal calf serum); EDTA (ethylene Diamine Tetraacetic Acid); EMT (epithelial-to-mesenchymal transition); TPX2 (Targeting protein for Xenopus kinesin-like protein 2).

\section{Acknowledgments}

This research was partly supported by Sun Hongbin for language revision.

\section{Disclosure Statement}

There are no conflicts among authors. 


\section{Cellular Physiology Cell Physiol Biochem 2015;36:2263-2273 \begin{tabular}{l|l} 
DOI: 10.1159/000430190 & (C) 2015 S. Karger AG, Basel
\end{tabular} www.karger.com/cpb \\ Niu et al.: Low Expression of miR-491 Promotes Cell Invasion}

\section{References}

1 Shigaki H, Baba Y, Watanabe M, Murata A, Ishimoto T, Iwatsuki M, Iwagami S, Nosho K, Baba H: Pik3ca mutation is associated with a favorable prognosis among patients with curatively resected esophageal squamous cell carcinoma. Clin Cancer Res 2013;19:2451-2459.

2 Zhang F, Yang Z, Cao M, Xu Y, Li J, Chen X, Gao Z, Xin J, Zhou S, Zhou Z, Yang Y, Sheng W, Zeng Y: Mir-203 suppresses tumor growth and invasion and down-regulates mir-21 expression through repressing ran in esophageal cancer. Cancer Lett 2014 ;342:121-129.

3 Esophageal cancer: Epidemiology, pathogenesis and prevention. Nat Clin Pract Gastr 2008;5:517-526.

4 Siegel R, Ma J, Zou Z, Jemal A: Cancer statistics, 2014. CA: Cancer J Clin 2014;64:9-29.

5 Mizushima T, Nakagawa H, Kamberov YG, Wilder EL, Klein PS, Rustgi AK: Wnt-1 but not epidermal growth factor induces beta-catenin/t-cell factor-dependent transcription in esophageal cancer cells. Cancer Res 2002;62:277-282.

6 Yang L, Leung AC, Ko JM, Lo PH, Tang JC, Srivastava G, Oshimura M, Stanbridge EJ, Daigo Y, Nakamura Y, Tang CM, Lau KW, Law S, Lung ML: Tumor suppressive role of a 2.4 mb 9q33-q34 critical region and dec1 in esophageal squamous cell carcinoma. Oncogene 2005;24:697-705.

7 Calin GA, Croce CM: Microrna signatures in human cancers. Nat Rev Cancer 2006;6:857-866.

8 Ambros V: The functions of animal micrornas. Nature 2004;431:350-355.

9 Ni Y, Meng L, Wang L, Dong W, Shen H, Wang G, Liu Q, Du J: Microrna-143 functions as a tumor suppressor in human esophageal squamous cell carcinoma. Gene 2013;517:197-204.

10 Zhang J, Cheng C, Yuan X, He JT, Pan QH, Sun FY: Microrna-155 acts as an oncogene by targeting the tumor protein 53-induced nuclear protein 1 in esophageal squamous cell carcinoma. Int J Clin Exp PAthol 2014;7:602-610.

11 Nakano H, Miyazawa T, Kinoshita K, Yamada Y, Yoshida T: Functional screening identifies a microrna, mir491 that induces apoptosis by targeting bcl-x(l) in colorectal cancer cells. Int J Cancer 2010;127:10721080.

12 Zhou Y, Li Y, Ye J, Jiang R, Yan H, Yang X, Liu Q Zhang J: Microrna-491 is involved in metastasis of hepatocellular carcinoma by inhibitions of matrix metalloproteinase and epithelial to mesenchymal transition. Liver Int 2013;33:1271-1280.

13 Liu SG, Qin XG, Zhao BS, Qi B, Yao WJ, Wang TY, Li HC, Wu XN: Differential expression of mirnas in esophageal cancer tissue. Oncol Lett 2013;5:1639-1642.

14 Heidebrecht HJ, Buck F, Steinmann J, Sprenger R, Wacker HH, Parwaresch R: P100: A novel proliferationassociated nuclear protein specifically restricted to cell cycle phases s, g2, and m. Blood 1997;90:226-233.

15 Liu Q, Yang P, Tu K, Zhang H, Zheng X, Yao Y: Tpx2 knockdown suppressed hepatocellular carcinoma cell invasion via inactivating akt signaling and inhibiting mmp2 and mmp9 expression. Chinese J Cancer Res 2014;26:410-417.

16 Jiang P, Shen K, Wang X, Song H, Yue Y, Liu T: Tpx2 regulates tumor growth in human cervical carcinoma cells. Mol Med Rep 2014;9:2347-2351.

17 Liu HC, Zhang GH, Liu YH, Wang P, Ma JF, Su LS, Li SL, Zhang L, Liu JW: Tpx2 sirna regulates growth and invasion of esophageal cancer cells. Biomed Pharmacother 2014;68:833-839.

18 Liu HC, Zhang Y, Wang XL, Qin WS, Liu YH, Zhang L, Zhu CL: Upregulation of the tpx2 gene is associated with enhanced tumor malignance of esophageal squamous cell carcinoma. Biomed Pharmacother 2013;67:751-755.

19 Hsu PK, Chen HY, Yeh YC, Yen CC, Wu YC, Hsu CP, Hsu WH, Chou TY: Tpx2 expression is associated with cell proliferation and patient outcome in esophageal squamous cell carcinoma. J Gastroenterol 2014;49:12311240.

20 Li YJ, Zhou JH, Du XX, Jia de X, Wu CL, Huang P, Han Y, Sui H, Wei XL, Liu L, Yuan HH, Zhang TT, Zhang WJ, Xie R, Lang XH, Liu T, Jiang CL, Wang LY, Bai YX: Dihydroartemisinin accentuates the anti-tumor effects of photodynamic therapy via inactivation of nf-kappab in eca109 and ec9706 esophageal cancer cells. Cell Physiol Biochem 2014;33:1527-1536.

21 Akanuma N, Hoshino I, Akutsu Y, Murakami K, Isozaki Y, Maruyama T, Yusup G, Qin W, Toyozumi T, Takahashi M, Suito H, Hu X, Sekino N, Matsubara H: Microrna-133a regulates the mrnas of two invadopodia-related proteins, fscn1 and mmp14, in esophageal cancer. Br J Cancer 2014;110:189-198. 


\section{Cellular Physiology Cell Physiol Biochem 2015;36:2263-2273 \begin{tabular}{l|l|l|}
\cline { 2 - 2 } DOI: 10.1159/000430190 & O 2015 S. Karger AG, Basel \\
\hline
\end{tabular} www.karger.com/cpb \\ Niu et al.: Low Expression of miR-491 Promotes Cell Invasion}

22 Yu X, Li H, Jiang X, Guo L, Jiang W, Lu SH: Mir-203 inhibits the proliferation and self-renewal of esophageal cancer stem-like cells by suppressing stem renewal factor bmi-1. Stem Cells Dev 2014;23:576-585

23 Li W, Jiang G, Zhou J, Wang H, Gong Z, Zhang Z, Min K, Zhu H, Tan Y: Down-regulation of mir-140 induces emt and promotes invasion by targeting slug in esophageal cancer. Cell Physiol Biochem 2014;34:14661476.

24 Zhu Y, Xia Y, Niu H, Chen Y: Mir-16 induced the suppression of cell apoptosis while promote proliferation in esophageal squamous cell carcinoma. Cell Physiol Biochem 2014;33:1340-1348.

25 Wang Z, Qiao Q, Chen M, Li X, Liu C, Xie Z: Mir-625 down-regulation promotes proliferation and invasion in esophageal cancer by targeting sox2. FEBS lett 2014;588:915-921.

26 Bartel DP: Micrornas: Genomics, biogenesis, mechanism, and function. Cell 2004;116:281-297.

27 Esquela-Kerscher A, Slack FJ: Oncomirs - micrornas with a role in cancer. Nat Rev Cancer 2006;6:259-269.

28 Rabinowits G, Gercel-Taylor C, Day JM, Taylor DD, Kloecker GH: Exosomal microrna: A diagnostic marker for lung cancer. Clin Lung Cancer 2009;10:42-46.

29 Mitchell PS, Parkin RK, Kroh EM, Fritz BR, Wyman SK, Pogosova-Agadjanyan EL, Peterson A, Noteboom J, O’Briant KC, Allen A, Lin DW, Urban N, Drescher CW, Knudsen BS, Stirewalt DL, Gentleman R, Vessella RL, Nelson PS, Martin DB, Tewari M: Circulating micrornas as stable blood-based markers for cancer detection. Proc Natl Acad Sci USA 2008;105:10513-10518.

30 Guo R, Wang Y, Shi WY, Liu B, Hou SQ Liu L: Microrna mir-491-5p targeting both tp53 and bcl-xl induces cell apoptosis in sw1990 pancreatic cancer cells through mitochondria mediated pathway. Molecules 2012;17:14733-14747.

31 Huang WC, Chan SH, Jang TH, Chang JW, Ko YC, Yen TC, Chiang SL, Chiang WF, Shieh TY, Liao CT, Juang JL, Wang HC, Cheng AJ, Lu YC, Wang LH: Mirna-491-5p and git1 serve as modulators and biomarkers for oral squamous cell carcinoma invasion and metastasis. Cancer Res 2014;74:751-764.

32 Yuan XW, Wang DM, Hu Y, Tang YN, Shi WW, Guo XJ, Song JG: Hepatocyte nuclear factor 6 suppresses the migration and invasive growth of lung cancer cells through p53 and the inhibition of epithelialmesenchymal transition. J Biol Chem 2013;288:31206-31216.

33 Ye J, Wu D, Shen J, Wu P, Ni C, Chen J, Zhao J, Zhang T, Wang X, Huang J: Enrichment of colorectal cancer stem cells through epithelial-mesenchymal transition via cdh1 knockdown. Mol Med Rep 2012;6:507-512.

34 Shi L, Jackstadt R, Siemens H, Li H, Kirchner T, Hermeking H: P53-induced mir-15a/16-1 and ap4 form a double-negative feedback loop to regulate epithelial-mesenchymal transition and metastasis in colorectal cancer. Cancer Res $2014 ; 74: 532-542$.

35 Kitamura K, Seike M, Okano T, Matsuda K, Miyanaga A, Mizutani H, Noro R, Minegishi Y, Kubota K, Gemma A: Mir-134/487b/655 cluster regulates tgf-beta-induced epithelial-mesenchymal transition and drug resistance to gefitinib by targeting magi2 in lung adenocarcinoma cells. Mol Cancer Ther 2014;13:444-453.

36 Paterson EL, Kazenwadel J, Bert AG, Khew-Goodall Y, Ruszkiewicz A, Goodall GJ: Down-regulation of the mirna-200 family at the invasive front of colorectal cancers with degraded basement membrane indicates emt is involved in cancer progression. Neoplasia 2013;15:180-191.

37 Ouyang H, Gore J, Deitz S, Korc M: Microrna-10b enhances pancreatic cancer cell invasion by suppressing tip30 expression and promoting egf and tgf-beta actions. Oncogene 2014;33:4664-4674. 